\title{
Low Dose Characterization of Diamondoid Carbon Nanothreads by Transmission Electron Microscopy
}

Stephen Juhl ${ }^{1}$, Xiang $\mathrm{Li}^{1}$, John Badding ${ }^{1,2}$, and Nasim Alem ${ }^{2}$

${ }^{1}$ Department of Chemistry, Pennsylvania State University, University Park, PA, USA

${ }^{2}$ Department of Materials Science and Engineering, Pennsylvania State University, University Park, PA, USA

Soft materials present a difficult case for characterization in the transmission electron microscope (TEM). Due to the use of high energy electrons, samples degrade rapidly from knock-on damage and radiolysis. Still, the atomic-scale structural and chemical information that can be gained from the TEM are attractive for newly discovered materials with nanoscale crystallinity. Diamondoid carbon nanothreads are one such material, which has been produced by compressing benzene to extremely high pressures in a diamond anvil cell.[1] X-ray diffraction data shows the nanothreads consist of cylindrically-symmetric columns of charge that are packed in a two-dimensional hexagonal lattice.[1] However, pair-distribution function analysis shows that the axial order extends to only $15 \mathrm{~nm}$. Therefore, it is ideal to probe the atomic and chemical structure of diamondoid carbon nanothreads through a battery of low-dose TEM techniques.

In this study, electron diffraction, electron energy loss spectroscopy (EELS), and high-resolution imaging are employed with reduced electron dose to investigate the atomic and chemical structure of nanothreads with minimal damage. Electron diffraction patterns in show a crystalline two-dimensional hexagonal lattice with lattice parameter $6.5 \AA$, as previously shown.[1] However, diffraction patterns from the sideon perspective show no off-axis diffraction, suggesting a lack of long-range atomic registry. Highresolution imaging of the nanothreads was complicated by degradation of the sample under a larger electron dose. Therefore, the critical dose is investigated by electron diffraction and EELS. Electron diffraction patterns in Figure 1a show a critical electron dose of $150 \mathrm{e}-/ \AA^{2}$ and expansion the lattice of the nanothread lattice. EELS spectra in Figure $1 \mathrm{~b}$ also reveal a rapid transformation of $\mathrm{sp}^{3}$ to $\mathrm{sp}^{2}$ carbon.[2] In this research, we quantify the $\mathrm{sp}^{2}$ ratio of the carbon present in the nanothreads using low dose spectroscopy techniques. In addition, we acquire low dose high resolution imaging by adjusting an electron gun monochromator to reduce the electron dose and minimize beam irradiation and damage while imaging the nanothreads on a high-resolution aberration-corrected transmission electron microscope.[3] High-resolution TEM images in Figure 1c and 1d show the transition from thread-like to amorphous morphology during exposure to high electron dose as an example. This study further analyzes low-dose images of nanothreads in order to compare their fine features with predicted nanothread structures. 
References:

[1] T. C. Fitzgibbons, M. Guthrie, E. Xu, V. H. Crespi, S. K. Davidowski, G. D. Cody, N. Alem, and J. V. Badding, "Benzene-derived carbon nanothreads," Nat. Mater., no. September, pp. 1-5, Sep. 2014.

[2] L. Ponsonnet, C. Donnet, K. Varlot, J. . M. Martin, a Grill, and V. Patel, "EELS analysis of hydrogenated diamond-like carbon films," Thin Solid Films, vol. 319, pp. 97-100, 1998.

[3] B. Barton, B. Jiang, C. Song, P. Specht, H. Calderon, and C. Kisielowski, "Atomic Resolution Phase Contrast Imaging and In-Line Holography Using Variable Voltage and Dose Rate," Microsc. Microanal., vol. 18, no. 5, pp. 982-994, 2012.
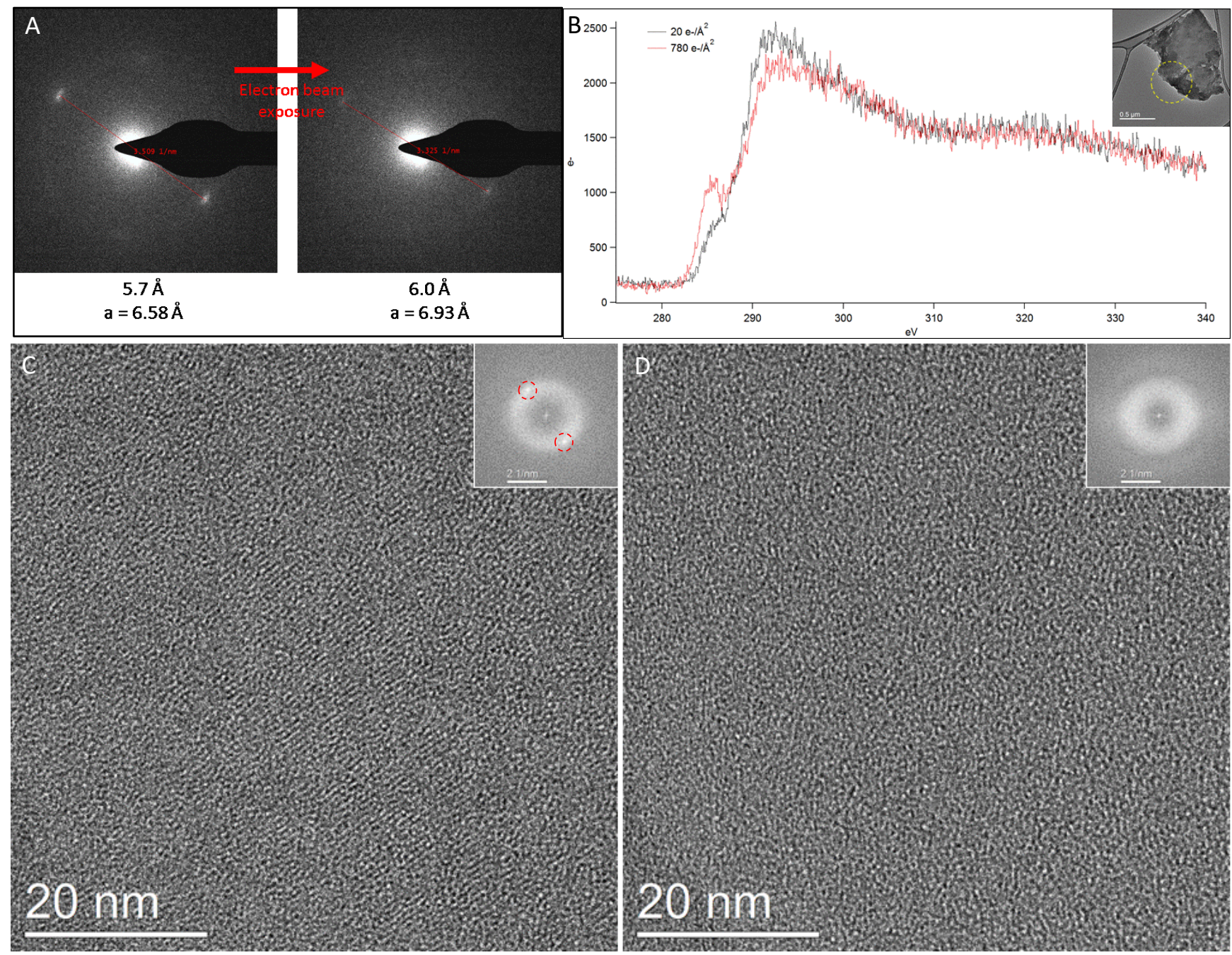

Figure 1. Electron diffraction patterns (A) show the effects of beam damage on diamondoid nanothreads, including expansion of the lattice and loss of crystallinity. EELS spectra (B) taken with a low electron dose show the increase in $\mathrm{sp}^{2}$ carbon content as the electron dose increases. Low-dose HREM images of nanothreads show thread-like features at a low electron dose (C) and amorphous features after elongated beam exposure (D). 